\title{
Proinsulin-to-C-peptide ratio versus proinsulin-to-insulin ratio in the prediction of incident diabetes: the Insulin Resistance Atherosclerosis Study (IRAS)
}

\author{
R. C. Loopstra-Masters • S. M. Haffner • C. Lorenzo • \\ L. E. Wagenknecht • A. J. Hanley
}

Received: 12 April 2011 / Accepted: 5 September 2011 /Published online: 30 September 2011

(C) Springer-Verlag 2011

\begin{abstract}
Aims Associations of proinsulin-to-insulin ratios with incident type 2 diabetes have been inconsistent. The use of Cpeptide as the denominator in the ratio may allow for better prediction because $\mathrm{C}$-peptide concentration is not affected by hepatic insulin clearance. The objective of this paper was to compare fasting intact and split proinsulin-to-insulin ratios (PI/I, SPI/I) with intact and split proinsulin-to-C-peptide ratios (PI/C-pep, SPI/C-pep) in the prediction of type 2 diabetes. Methods Prospective data on 818 multi-ethnic adults without diabetes at baseline from the Insulin Resistance Atherosclerosis Study (IRAS) were used. Insulin sensitivity $\left(\mathrm{S}_{\mathrm{I}}\right)$ and acute insulin response (AIR) were determined from frequently sampled intravenous glucose tolerance tests, and fasting intact and split proinsulin were measured using
\end{abstract}

Electronic supplementary material The online version of this article (doi:10.1007/s00125-011-2322-2) contains peer-reviewed but unedited supplementary material, which is available to authorised users.

R. C. Loopstra-Masters · A. J. Hanley $(\bowtie)$

Department of Nutritional Sciences, Faculty of Medicine,

University of Toronto,

FitzGerald Building, 150 College St,

Toronto, ON, Canada M5S 3E2

e-mail: anthony.hanley@utoronto.ca

S. M. Haffner

Department of Medicine, Baylor College of Medicine,

Houston, TX, USA

C. Lorenzo

Division of Clinical Epidemiology,

University of Texas Health Sciences Center,

San Antonio, TX, USA

L. E. Wagenknecht

Division of Public Health Sciences,

Wake Forest University School of Medicine,

Winston-Salem, NC, USA specific two-site monoclonal antibody-based immunoradiometric assays. Associations of proinsulin ratios with type 2 diabetes were determined using logistic regression and differences in prediction were assessed by comparing areas under the receiver operating characteristic curve (AROCs). Results In logistic regression analyses, PI/C-pep and SPI/Cpep were more strongly associated with incident type 2 diabetes $(n=128)$ than $\mathrm{PI} / \mathrm{I}$ and SPI/I, and were significantly better predictors of diabetes in AROC analyses (PI/C-pep $=0.662$ vs $\mathrm{PI} / \mathrm{I}=0.603, p=0.02$; SPI $/ \mathrm{C}-\mathrm{pep}=0.690$ vs $\mathrm{SPI} / \mathrm{I}=0.631, p=$ 0.01). Both PI/C-pep and SPI/C-pep were associated with type 2 diabetes after adjustment for age, sex, ethnicity, waist circumference, impaired glucose tolerance, lipids and $\mathrm{S}_{\mathrm{I}}$. Both $\mathrm{PI} / \mathrm{C}$-pep and SPI/C-pep were significantly associated with incident type 2 diabetes in models that included AIR.

Conclusions Proinsulin-to-C-peptide ratios were stronger predictors of diabetes in comparison with proinsulin-toinsulin ratios. These findings support the use of C-peptide as the denominator for proinsulin ratios, to more accurately reflect the degree of disproportional hyperproinsulinaemia.

Keywords Beta cell function - Insulin sensitivity ·

Proinsulin · Type 2 diabetes mellitus

\begin{tabular}{|c|c|}
\hline \multicolumn{2}{|c|}{ Abbreviations } \\
\hline AIR & Acute insulin response \\
\hline AROC & $\begin{array}{l}\text { Area under the receiver operating characteristic } \\
\text { curve }\end{array}$ \\
\hline FSIGT & $\begin{array}{l}\text { Frequently sampled intravenous glucose tol- } \\
\text { erance test }\end{array}$ \\
\hline IGT & Impaired glucose tolerance \\
\hline IRAS & Insulin Resistance Atherosclerosis Study \\
\hline NGT & Normal glucose tolerance \\
\hline PI/C-pep & Intact proinsulin-to-C-peptide ratio \\
\hline $\mathrm{PI} / \mathrm{I}$ & Intact proinsulin-to-insulin ratio \\
\hline
\end{tabular}


$\mathrm{S}_{\mathrm{I}} \quad$ Insulin sensitivity

SPI/C-pep Split proinsulin-to-C-peptide ratio

SPI/I Split proinsulin-to-insulin ratio

\section{Introduction}

Type 2 diabetes is characterised by higher circulating concentrations of intact and split proinsulin, in absolute amounts and as proportions of total insulin secretory products [1-3]. Although the cause of disproportional hyperproinsulinaemia in diabetes is unknown, it may arise from inefficient proinsulin processing within the beta cell secretory granule or the premature release of proinsulin as a result of increased demand for insulin in insulin-resistant states [4]. Elevations in the ratio of proinsulin to insulin as well as absolute proinsulin concentrations adjusted for fasting insulin have been proposed as early markers of beta cell dysfunction [5]. Prospective observational studies have reported significant associations of total, intact and split proinsulin-to-insulin ratios with incident type 2 diabetes [4, 6-9]; proinsulin concentrations adjusted for fasting insulin have also been related to incident diabetes [10-13]. However, other studies have reported non-significant associations of proinsulin-to-insulin ratios with incident type 2 diabetes (although it should be noted that proinsulin itself was predictive in these studies) [13, 14].

One possible explanation for these inconsistent findings is that the use of fasting insulin as the denominator in proinsulin ratios may not be the optimal method for documenting relative hyperproinsulinaemia, as fasting insulin concentrations are affected by both beta cell secretion and hepatic clearance of insulin [15]. Hepatic insulin clearance is reduced in insulinresistant individuals [16], which contributes to elevated fasting insulin concentrations in these participants and would result in a deflation in the proinsulin-to-insulin ratio. Vauhkonen et al. suggested that proinsulin-to-C-peptide ratios may be better indicators of distressed beta cells as $\mathrm{C}$ peptide is not cleared by the liver, and therefore concentrations are less affected by insulin resistance [15].

The majority of observational studies that have examined the relationship between proinsulin ratios and incident diabetes have used fasting insulin concentrations as the denominator [4, 6-14]. To our knowledge, no studies have compared proinsulin-to-C-peptide ratios with proinsulin-toinsulin ratios in the prediction of type 2 diabetes. Further, it is not clear whether disproportional hyperproinsulinaemia reflects the same dimension of beta cell dysfunction as other secretory markers (such as those derived from stimulated protocols). A limited number of studies have shown proinsulin, adjusted for fasting insulin, to be independently related to type 2 diabetes after adjustment for other markers of beta cell function, specifically acute insulin response
(AIR) $[10,11]$ and early insulin response [12], but no studies have looked at the ability of proinsulin-to-C-peptide ratio to predict diabetes independent of these measures.

Our study objectives included examining the associations of proinsulin-to-insulin and proinsulin-to-C-peptide ratios with incident diabetes in a multi-ethnic population with detailed measures of glucose tolerance status, insulin sensitivity $\left(\mathrm{S}_{\mathrm{I}}\right)$ and AIR.

\section{Methods}

A detailed methodology for the Insulin Resistance Atherosclerosis Study (IRAS) has been published [10, 17]. The study aimed to include equal numbers of non-Hispanic white, African-American and Hispanic adults with normal glucose tolerance (NGT), impaired glucose tolerance (IGT) and type 2 diabetes. The baseline population included 1,625 participants. Follow-up study visits occurred approximately 5 years after baseline. Study protocols, which included measures of waist circumference, height, weight and selfreported ethnicity, were identical for baseline and follow-up and were obtained by standard measures [17]. All participants gave written informed consent and the institutional review boards at each study centre provided ethical approval.

For the purposes of the current study, 559 participants with diabetes at baseline were excluded. In addition, participants who did not return for follow-up study visits $(n=175)$ were excluded, as were participants with missing data for fasting insulin and proinsulin concentrations and/or AIR or $\mathrm{S}_{\mathrm{I}}$. The resulting study population for the current analysis was 818 .

Blood samples were collected after participants had fasted for $12 \mathrm{~h}$ and abstained from heavy exercise, alcohol and smoking before the study visit [17]. A standard OGTT and modified frequently sampled intravenous glucose tolerance test (FSIGT) were performed on two study days separated by 1 week at minimum, 1 month at maximum [17]. The validated modified FSIGT protocol included an injection of regular insulin, rather than tolbutamide, and a reduced number of samples [17]. Data from the FSIGT were used to calculate $\mathrm{S}_{\mathrm{I}}$, using MINMOD mathematical modelling analysis (version 3.0, 1994), and AIR, which was calculated as the mean plasma insulin concentration from 2 and $4 \mathrm{~min}$ time points after the initial administration of glucose.

Laboratory analyses of glucose, insulin and C-peptide took place at the University of Southern California (Los Angeles, CA, USA), where plasma samples were shipped on a monthly basis. The glucose oxidase technique on an automated autoanalyser (Yellow Springs Equipment, Yellow Springs, OH, USA) was used to analyse plasma glucose concentrations. Insulin was measured by a dextran-charcoal RIA that cross-reacted with proinsulin. The CV for the insulin RIA based on assays of 163 split- 
pairs was $19 \%$. C-peptide was measured using the NOVONordisk human C-peptide method (NOVO-Nordisk, Copenhagen, Denmark). The intra-assay $\mathrm{CV}$ was $3.2 \%$ and the inter-assay $\mathrm{CV}$ was $16.5 \%$.

Fasting serum concentrations of intact and split proinsulin were measured in the laboratory of the Department of Clinical Biochemistry at Addenbrooke's Hospital (Cambridge, UK) after an average of 3.3 years of storage at $-70^{\circ} \mathrm{C}$. Two highly specific two-site monoclonal antibody-based immunoradiometric assays were used to measure intact and split proinsulin. The assay for intact proinsulin had no detectable cross-reactivity with insulin or split proinsulin and its split-pair CV was $14 \%(n=98)$. The split proinsulin assay cross-reacted equally with 32,33 , des-32 and des-31, 32 split proinsulins, the major component being des-31, 32; however, it did not cross-react with insulin. The cross-reactivity with intact proinsulin was $84 \%$, so assay values of split proinsulin were corrected by subtracting corresponding proinsulin cross-reactivity. The split-pair CV for this assay was $18 \%$ for 94 split-pairs [10].

Incident cases of diabetes at follow-up were defined by fasting or $2 \mathrm{~h}$ postload glucose concentrations according 1999 WHO guidelines [18]. In addition, participants with a new recorded use of oral hypoglycaemic agents or insulin were considered to have incident diabetes.

Statistical analysis Intact and split proinsulin concentrations were divided by fasting $\mathrm{C}$-peptide and insulin concentrations to yield intact and split proinsulin-to-Cpeptide ratios (PI/C-pep, SPI/C-pep) as well as intact and split proinsulin-to-insulin ratios (PI/I, SPI/I). These variables were log-transformed because they displayed nonnormal distributions that were improved by natural log transformation. Similarly, AIR was log-transformed. Characteristics of participants who converted to type 2 diabetes in comparison with those who did not were presented as means and SD and tested for differences by non-parametric Kruskal-Wallis tests. Categorical variables were presented as $n(\%)$ and tested for differences by $\chi^{2}$ analyses.

Multivariable logistic regression analysis was used to assess risk of incident diabetes per 1-SD increase in the natural $\log$ ratios of PI/I, SPI/I, PI/C-pep and SPI/C-pep. The base model included adjustment for sex, ethnicity and age; a second model was additionally adjusted for waist circumference, triacylglycerol, HDL-cholesterol and glucose tolerance status, and the fully adjusted model was adjusted for $S_{I}$. Sensitivity analyses were carried out substituting BMI for waist circumference (data not shown). Since there was little difference, the models with waist circumference are reported here. In addition to analyses in the full population, an a priori decision was made to conduct the logistic regression analyses in the study population stratified by baseline glucose tolerance as well as the median split of baseline $\mathrm{S}_{\mathrm{I}}$ and AIR. We also tested whether associations between proinsulin ratios were independent of AIR. Interaction analyses using categorical values defined by quartiles of the proinsulin ratios were conducted for ethnicity and sex.

To evaluate the ability of PI/C-pep and SPI/C-pep ratios to predict risk of type 2 diabetes relative to PI/I and SPI/I ratios, comparisons of the areas under the receiver operating characteristic curve (AROCs) were performed, with significance determined by the DeLong algorithm [19]. Comparisons were made using data from minimally adjusted logistic regression models.

In light of the potential of ORs to overestimate the relative risk when the incidence of the outcome exceeds $10 \%$, we re-ran the analyses for the full population using Poisson regression. The magnitude, direction and significance of the findings were very similar to those from the logistic regression models (data not shown).

\section{Results}

A total of 128 out of 818 participants were found to have developed diabetes after an average of 5.2 years of followup, a cumulative incidence rate of $15.7 \%$ (Table 1). Those who converted to type 2 diabetes were significantly older, were more likely to have had IGT, and had significantly higher waist circumference and BMI values at baseline. In addition, those who converted had lower baseline $S_{I}$ and AIR values and higher fasting insulin and $C$-peptide concentrations. All proinsulin ratios at baseline, including PI/C-pep, SPI/C-pep, PI/I and SPI/I, were significantly higher amongst those who converted to type 2 diabetes.

In multivariable logistic regression analyses, PI/C-pep and SPI/C-pep ratios were more strongly associated with incident diabetes than the corresponding PI/I and SPI/I ratios (Table 2) and were significantly better predictors of type 2 diabetes in AROC analyses (PI/C-pep=0.662, PI/I= 0.603, $p=0.02$; SPI $/ \mathrm{C}$-pep $=0.690, \mathrm{SPI} / \mathrm{I}=0.631, p=0.01$ ). Both PI/C-pep and SPI/C-pep ratios were significantly associated with an increased risk of type 2 diabetes in the whole study population and associations remained strong in the fully adjusted models. The PI/I ratio was not found to be significantly related to an increased risk of diabetes after adjustment for waist circumference, triacylglycerol, HDLcholesterol, glucose tolerance and $\mathrm{S}_{\mathrm{I}}$ (model 3: OR 1.17 [95\% CI 0.96, 1.45]). The SPI/I ratio was related to an increased risk of diabetes in all models, although risk estimates were lower than those observed for the SPI/C-pep ratio (Table 2 ).

When the multivariable logistic regression analyses were carried out separately for NGT and IGT participants, PI/Cpep and SPI/C-pep ratios remained strongly related to an 
Table 1 Subject characteristics for study population and by conversion to type 2 diabetes $(n=818)$

\begin{tabular}{|c|c|c|c|c|}
\hline Characteristic & Total population & Non-converters & Converters to type 2 diabetes & $p$ value \\
\hline$n(\%)$ & $818(100)$ & $690(84.4)$ & $128(15.7)$ & - \\
\hline Age (years) & $54.7(8.5)$ & $54.4(8.5)$ & $56.5(8.1)$ & 0.0099 \\
\hline Males, $n(\%)$ & $360(44.0)$ & $308(44.6)$ & $52(40.6)$ & 0.4009 \\
\hline Ethnicity, $n(\%)$ & & & & 0.6497 \\
\hline Non-Hispanic white & $330(40.3)$ & $280(40.6)$ & $50(39.1)$ & \\
\hline African-American & $203(24.8)$ & $174(25.2)$ & $29(22.7)$ & \\
\hline Hispanic & $285(34.8)$ & $236(34.2)$ & $49(38.3)$ & \\
\hline Glucose tolerance status, $n(\%)$ & & & & $<0.0001$ \\
\hline Normal & $552(67.5)$ & $508(73.6)$ & $44(34.4)$ & \\
\hline IGT & $266(32.5)$ & $182(26.4)$ & $84(65.6)$ & \\
\hline Waist circumference $(\mathrm{cm})$ & $90.3(12.5)$ & $89.4(12.4)$ & $95.2(12.0)$ & $<0.0001$ \\
\hline BMI $\left(\mathrm{kg} / \mathrm{m}^{2}\right)$ & $28.3(5.5)$ & $27.9(5.3)$ & $30.8(6.1)$ & $<0.0001$ \\
\hline Fasting insulin (pmol/1) & $108.5(105.2)$ & $98.8(74.6)$ & $160.5(194.5)$ & $<0.0001$ \\
\hline Fasting C-peptide (nmol/l) & $0.56(0.25)$ & $0.54(0.24)$ & $0.69(0.27)$ & $<0.0001$ \\
\hline Fasting intact proinsulin (pmol/1) & $5.9(5.3)$ & $5.4(4.7)$ & $9.1(6.9)$ & $<0.0001$ \\
\hline Fasting split proinsulin (pmol/l) & $8.0(7.5)$ & $7.0(6.2)$ & $13.4(11.0)$ & $<0.0001$ \\
\hline $\mathrm{S}_{\mathrm{I}}\left(\times 10^{-4} \min ^{-1}[\mu \mathrm{U} / \mathrm{ml}]^{-1}\right)^{\mathrm{a}}$ & $2.21(2.04)$ & $2.37(2.07)$ & $1.36(1.62)$ & $<0.0001$ \\
\hline $\operatorname{AIR}(\mu \mathrm{U} / \mathrm{ml})^{\mathrm{b}}$ & $67.3(57.4)$ & $70.6(59.7)$ & $49.5(37.6)$ & $<0.0001$ \\
\hline PI/C-pep & $10.8(7.3)$ & $10.3(7.2)$ & $13.2(7.4)$ & $<0.0001$ \\
\hline SPI/C-pep & $13.6(9.5)$ & $12.8(9.0)$ & $18.2(10.8)$ & $<0.0001$ \\
\hline $\mathrm{PI} / \mathrm{I}$ & $0.066(0.051)$ & $0.064(0.049)$ & $0.075(0.062)$ & 0.0055 \\
\hline SPI/I & $0.082(0.067)$ & $0.079(0.064)$ & $0.101(0.076)$ & 0.0002 \\
\hline
\end{tabular}

Data are presented as mean (SD) unless otherwise stated

$p$ values for $\chi^{2}$ values or Kruskal-Wallis tests for a comparison between non-converters and converters

${ }^{\mathrm{a}}$ To convert values to SI units $\left(\mu \mathrm{mol} \mathrm{m}{ }^{-2} \min ^{-1}\right.$ (pmol/1) ${ }^{-1}$ ) multiply by 0.167

${ }^{\mathrm{b}}$ To convert values to SI units (pmol/l) multiply by 6.945

increased risk of diabetes in both groups, although risk estimates were lower amongst IGT participants. The OR for a 1-SD increase in natural $\log \mathrm{PI} / \mathrm{C}$-pep ratio in the fully adjusted model was $1.62(95 \%$ CI $1.19,2.20)$ in NGT participants and $1.44(1.07,1.93)$ in IGT participants (Fig. 1a). The PI/I ratio was not significantly related to an increased risk of diabetes amongst NGT or IGT participants. The ORs in the fully adjusted models were $1.31(95 \%$ CI $0.94,1.82)$ and 1.15 (95\% CI $0.91,1.57)$ for NGT and IGT participants, respectively. SPI/C-pep was associated with an increased risk of diabetes in both NGT and IGT groups, with an OR of $1.70(95 \%$ CI $1.23,2.36)$ amongst NGT participants and 1.50 (95\% CI $1.09,2.06$ ) amongst IGT participants (Fig. 1a). SPI/I was borderline significantly related to a higher risk of diabetes in the NGT group (OR 1.39 [95\% CI 1.00, 1.93], Model 3) but was not significantly related to incident diabetes amongst IGT participants (OR 1.16 [95\% CI $0.87,1.54]$ ) (Fig. 1a).

Differences in the predictive abilities of both PI/Cpep and SPI/C-pep ratios were observed between median-split $\mathrm{S}_{\mathrm{I}}$ groups. In the group with $\mathrm{S}_{\mathrm{I}}$ values below the median $\left(<1.65 \times 10^{-4} \mathrm{~min}^{-1}\left[\mu \mathrm{U} / \mathrm{ml}^{-1}\right.\right.$; to convert values to SI units $\left[\mu \mathrm{mol} \mathrm{m}{ }^{-2} \mathrm{~min}^{-1} \text { (pmol/l) }\right)^{-1}$ ] multiply by 0.167$), \mathrm{PI} / \mathrm{C}$-pep and SPI/C-pep ratios were strongly significantly related to an increased risk of diabetes (Fig. 1b). Amongst participants with high $\mathrm{S}_{\mathrm{I}}$ values $\left(\geq 1.65 \times 10^{-4} \mathrm{~min}^{-1}[\mu \mathrm{U} / \mathrm{ml}]^{-1}\right)$, there was no significant relationship between $\mathrm{PI} / \mathrm{C}$-pep or SPI/C-pep ratio and incident diabetes. Proinsulin-to-insulin ratios were not significantly related to diabetes in either $\mathrm{S}_{\mathrm{I}}$ group. Interestingly, the ORs for both proinsulin-to-C-peptide and proinsulin-to-insulin ratios were in the same range (OR $\sim 1.30$ [95\% CI $\sim 0.90,1.90]$ ) amongst participants with greater $\mathrm{S}_{\mathrm{I}}$ values (Fig. 1b).

AROC analyses showed that, amongst participants with $\mathrm{S}_{\mathrm{I}}$ values below the median, PI/C-pep was a significantly better predictor of type 2 diabetes compared with PI/I (AROC 0.665 vs 0.601 , respectively, $p=0.03$ ). Amongst NGT and IGT participants, the discrepancy between the two ratios was not as strong (AROC 0.691 vs $0.637, p=0.059$ for NGT and AROC 0.663 vs $0.559, p=$ 
Table 2 Multivariable logistic regression analysis of baseline measures of proinsulin and risk of incident diabetes $(n=818)$

\begin{tabular}{llrr}
\hline Independent variable & OR $(95 \% \mathrm{CI})$ & Wald $\chi^{2}$ & $p$ value \\
\hline Log PI/C-pep & & & \\
Model 1 & $1.61(1.32,1.96)$ & 23.15 & $<0.0001$ \\
Model 2 & $1.51(1.22,1.86)$ & 14.14 & 0.0002 \\
Model 3 & $1.50(1.21,1.86)$ & 13.86 & 0.0002 \\
Log SPI/C-pep & & & \\
Model 1 & $1.94(1.57,2.40)$ & 36.74 & $<0.0001$ \\
Model 2 & $1.67(1.33,2.11)$ & 18.97 & $<0.0001$ \\
Model 3 & $1.65(1.30,2.09)$ & 17.43 & $<0.0001$ \\
Log PI/I & & & \\
Model 1 & $1.21(0.998,1.47)$ & 3.75 & 0.0529 \\
Model 2 & $1.13(0.92,1.39)$ & 1.36 & 0.2443 \\
Model 3 & $1.17(0.96,1.45)$ & 2.13 & 0.1445 \\
Log SPI/I & & & \\
Model 1 & $1.44(1.17,1.76)$ & 12.39 & 0.0004 \\
Model 2 & $1.22(0.99,1.52)$ & 3.50 & 0.0615 \\
Model 3 & $1.24(1.00,1.54)$ & 3.76 & 0.0525 \\
\hline
\end{tabular}

Model 1: Adjusted for age, sex and ethnicity

Model 2: Additionally adjusted for waist circumference, triacylglycerol, HDL-cholesterol and glucose tolerance status at baseline

Model 3: Additionally adjusted for $\mathrm{S}_{\mathrm{I}}$

OR refers to risk of diabetes per 1-SD increase in independent variable Natural log transformations were used

0.12 for IGT). There was no difference in predictive ability between PI/C-pep and PI/I amongst participants with high $\mathrm{S}_{\mathrm{I}}$ values (AROC 0.615 vs $0.623, p=0.67$ ). Similarly, SPI/Cpep was a better predictor of type 2 diabetes amongst participants with low $\mathrm{S}_{\mathrm{I}}$ values compared with SPI/I (AROC 0.678 vs $0.612, p=0.02)$, but there were no significant differences in prediction abilities in the glucose tolerance subgroups.

When AIR was added to multivariable logistic regression models in the full study population, PI/Cpep and SPI/C-pep ratios remained strongly independently related to an increased risk of type 2 diabetes, as did AIR (Table 3). The SPI/I ratio was attenuated by the addition of AIR to the logistic regression model to borderline significance (OR 1.25 [95\% CI 1.00, 1.55]) while the PI/I ratio was not related to incident diabetes in these models. In an analysis within subgroups of AIR (defined by the median split), PI/C-pep and SPI/C-pep ratios were independently related to incident type 2 diabetes in both groups, and the magnitudes of the ORs were stronger than those for PI/I and SPI/I in all models (Electronic supplementary material [ESM] Tables 1 and 2).

Interaction terms were not significant for any of the proinsulin ratios with ethnicity or sex.

\section{Discussion}

In the IRAS study population, we observed that both PI/Cpep and SPI/C-pep ratios were strong predictors of incident diabetes, irrespective of glucose tolerance status, and that these ratios were better predictors of incident diabetes than $\mathrm{PI} / \mathrm{I}$ and SPI/I ratios. The discrepancy in predictive ability was most profound in participants with lower $\mathrm{S}_{\mathrm{I}}$ values and was not apparent in participants with $\mathrm{S}_{\mathrm{I}}$ values above the median split, suggesting that insulin resistance affects the ability of proinsulin-to-insulin ratios to predict diabetes (with little difference between the ratios amongst insulinsensitive participants).

Our data support the suggestion by Vauhkonen et al. [15] that C-peptide be used as the denominator in proinsulin ratios to better estimate the relative proportion of proinsulin being secreted. This is because fasting insulin concentrations are affected by hepatic clearance of insulin, which has been observed to be reduced in insulin-resistant states, while C-peptide concentrations are not similarly affected. Thus, the use of fasting insulin as the denominator in proinsulin ratios may lead to diluted values as a result of elevations in fasting insulin concentrations caused by reduced hepatic clearance. Our observations that the proinsulin-to-insulin ratios were not associated with an increased risk of diabetes in participants with lower $\mathrm{S}_{\mathrm{I}}$ support these concerns about the use of proinsulin-toinsulin ratios for diabetes risk estimation.

Numerous studies have reported that proinsulin-toinsulin ratios are significantly related to increased risk of diabetes [4, 6-12], although inconsistencies have been reported [13, 14]. Specifically, Wareham et al. found that the ratio of proinsulin-to-insulin was not increased amongst IGT participants at baseline and that the ratio did not significantly predict diabetes after adjustment for age, sex and glucose tolerance status in the whole study population [14]. Nijpels et al. studied the relationship between proinsulin-to-insulin ratio in participants with IGT and reported that the ratio was not significantly related to an increased risk of diabetes in these participants [13]. These discrepancies in the literature could be due to differences across studies in the effect of insulin resistance on hepatic plasma insulin clearance and the resulting impact on fasting insulin concentrations. This point is of relevance to the studies mentioned above, as participants with IGT are known to have insulin resistance and hyperinsulinaemia.

In spite of the use of insulin as a marker of insulin secretion to evaluate disproportional hyperproinsulinaemia, a number of studies have reported significant relationships between these measures and incident diabetes [4, 7-12, 14], although it is likely that the magnitude of risk of type 2 diabetes was underestimated in these studies for the reasons mentioned above. Conversely, it is possible that there was a 
Fig. 1 a ORs for proinsulin ratios in the total study population and glucose tolerance subgroups. OR adjusted for age, sex, ethnicity, waist circumference, glucose tolerance status at baseline and insulin sensitivity and corresponds to a 1-SD increase in natural log proinsulin ratio. There were 128 events amongst 818 participants in the whole population, 44 events amongst 552 participants in the NGT subgroup and 84 events amongst 266 participants in the IGT subgroup. b ORs for proinsulin ratios in the total study population and insulin sensitivity subgroups. OR adjusted for age, sex, ethnicity, waist circumference and glucose tolerance status at baseline, and corresponds to a 1-SD increase in natural $\log$ proinsulin ratio. There were 128 events amongst 818 participants in the whole population, 97 events amongst 408 participants in the low $\mathrm{S}_{\mathrm{I}}$ subgroup and 31 events amongst 410 participants in the high $\mathrm{S}_{\mathrm{I}}$ subgroup a

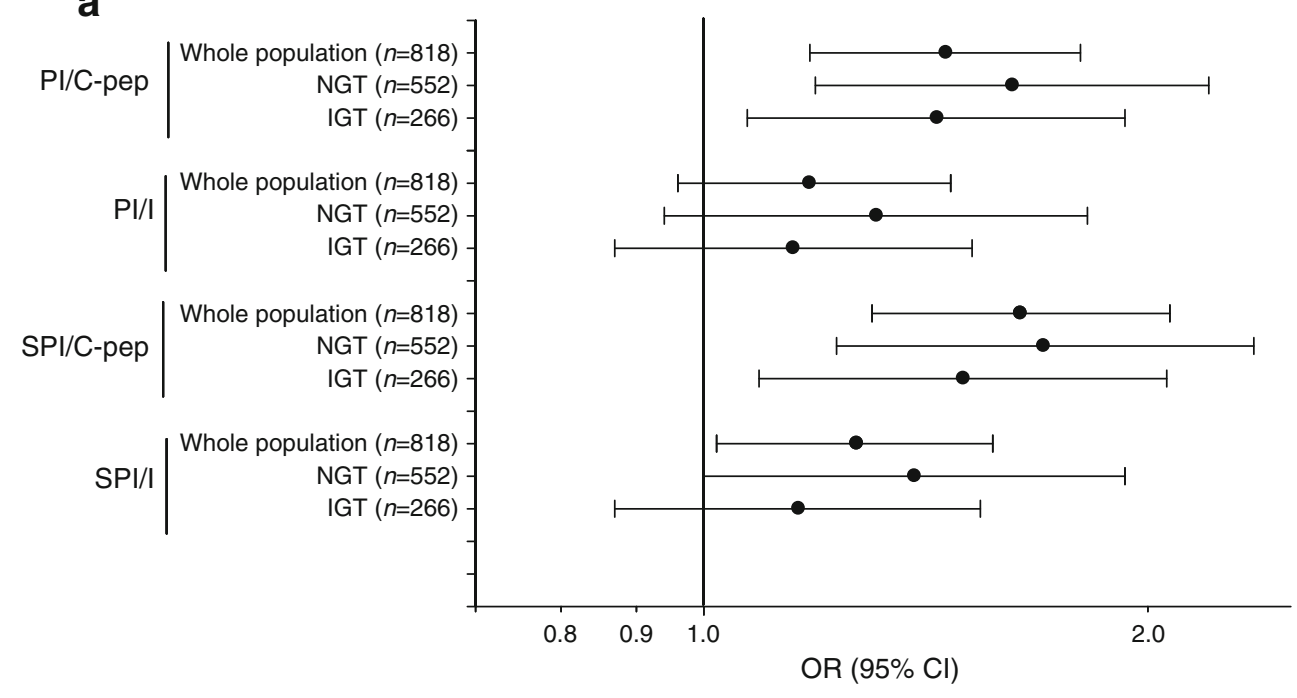

b

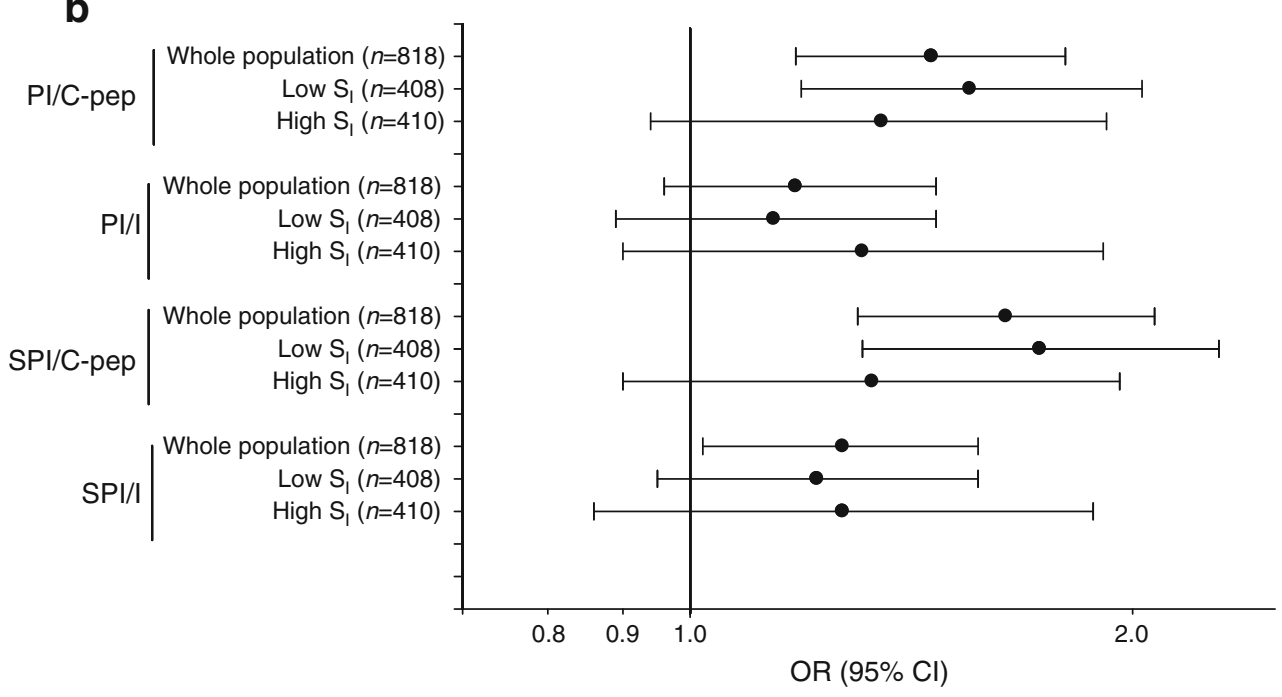

lower prevalence of insulin resistance in these study populations; however, this supposition cannot be verified as insulin sensitivity data were not reported in the majority of the related prospective studies [4, 6-11, 13, 14]. Zethelius et al. reported that proinsulin concentrations adjusted for insulin were related to incident diabetes independent of $\mathrm{S}_{\mathrm{I}}$ amongst elderly white men, but the analysis was not stratified by $\mathrm{S}_{\mathrm{I}}[12]$.

Our study also showed that proinsulin-to-C-peptide ratios were predictors of diabetes independent of AIR. These findings lend further support to the hypothesis that disproportional hyperproinsulinaemia may reflect a different dimension of beta cell function than is captured by AIR (e.g. disordered processing vs loss of beta cell mass) $[10,11]$.

The strengths of our study include the use of precise measures of AIR and $\mathrm{S}_{\mathrm{I}}$, the relatively large sample size and representation of three ethnic groups in the study population. In addition, measures of both intact and split proinsulin were available. Limitations include the use of data from a non-specific insulin assay, which displayed a high degree of cross-reactivity with proinsulin. Nonspecific insulin assays detect all circulating immunoreactive insulin-like materials. In non-diabetic participants, it has been demonstrated that proinsulin and its split products constitute only about $10 \%$ of this material. Since all participants were non-diabetic at baseline, this crossreactivity would have impacted all participants to the same general degree. In interpreting the results of the present study, it is important to bear in mind the longer halflife and lower inter-individual variability of C-peptide vs insulin. To address this issue, an examination of stimulated proinsulin-to-C-peptide ratios vs proinsulin-to-insulin ratios would have been ideal, as stimulated concentrations are thought to be a better reflection of beta cell secretory granule content and they are less affected by elimination kinetics [20]. 
Table 3 Multivariable logistic regression analyses of proinsulin ratios and AIR and risk of incident diabetes $(n=818)$

\begin{tabular}{llrr}
\hline Independent variable & OR $(95 \% \mathrm{CI})$ & Wald $\chi^{2}$ & $p$ value \\
\hline Log PI/C-pep & $1.46(1.17,1.82)$ & 11.57 & 0.0007 \\
Log AIR & $0.55(0.43,0.69)$ & 25.95 & $<0.0001$ \\
Log SPI/C-pep & $1.73(1.36,2.21)$ & 19.78 & $<0.0001$ \\
Log AIR & $0.52(0.41,0.66)$ & 29.12 & $<0.0001$ \\
Log PI/I & $1.12(0.91,1.38)$ & 1.11 & 0.2922 \\
Log AIR & $0.55(0.44,0.69)$ & 25.18 & $<0.0001$ \\
Log SPI/I & $1.25(1.00,1.55)$ & 3.98 & 0.0461 \\
Log AIR & $0.55(0.43,0.69)$ & 25.85 & $<0.0001$
\end{tabular}

The table shows the results of logistic regression models of the natural log of each of the proinsulin ratios with the natural log of AIR (i.e. four separate models), adjusted for age, sex, ethnicity, waist circumference, glucose tolerance status at baseline and insulin sensitivity

OR refers to relative change in odds of diabetes per 1-SD increase in independent variable

Natural $\log$ transformations were used

Notwithstanding, fasting proinsulin-to-insulin ratios have been correlated with acutely stimulated proinsulin-to-insulin ratios [21]. Given the lack of clear differences in prediction between intact and split proinsulin in the present analysis, the value of measuring intact and split proinsulin (vs total proinsulin) requires additional study. Further, statistical power was reduced in our subgroup analysis because of the relatively small number of events, particularly in our high $\mathrm{S}_{\mathrm{I}}$ group. In addition, the CVs of our RIA for insulin, C-peptide and intact and split proinsulin were high. Finally, in light of the high prevalence of IGT and insulin resistance in the study population, these findings need to be confirmed in larger general population studies.

In conclusion, it was observed that, in comparison with proinsulin-to-insulin ratios, proinsulin-to-C-peptide ratios were stronger predictors of incident diabetes in a multi-ethnic study population, and that the differences in predictive ability were most profound in participants with low $\mathrm{S}_{\mathrm{I}}$. These findings strongly support the use of Cpeptide as a ratio denominator to more accurately reflect the degree of disproportional proinsulin being secreted by beta cells.

Acknowledgements A.J. Hanley holds a Tier II Canada Research Chair in Diabetes Epidemiology. The IRAS was supported by National Heart, Lung and Blood Institute grants U01-HL47887, U01-HL47889, U01-HL47892, U01-HL47902, DK-29867 and R01 58329 and grant M01-RR-43 from the National Institutes of Health.

Contribution statement All authors contributed to the conception and design of the study, analysis and interpretation of data, and drafting the article or revising it critically for important intellectual content. All authors have read and approved the final version of the article.
Duality of interest The authors declare that there is no duality of interest associated with this manuscript.

\section{References}

1. Tura A, Pacini G, Kautzky-Willer A, Ludvik B, Prager R, Thomaseth K (2003) Basal and dynamic proinsulin-insulin relationship to assess beta-cell function during OGTT in metabolic disorders. Am J Physiol Endocrinol Metab 285: E155-E162

2. Røder ME, Porte D Jr, Schwartz RS, Kahn SE (1998) Disproportionately elevated proinsulin levels reflect the degree of impaired B cell secretory capacity in patients with noninsulin-dependent diabetes mellitus. J Clin Endocrinol Metab 83:604-608

3. Saad MF, Kahn SE, Nelson RG et al (1990) Disproportionately elevated proinsulin in Pima Indians with noninsulin-dependent diabetes mellitus. J Clin Endocrinol Metab 70:1247-1253

4. Haffner SM, Gonzalez C, Mykkanen L, Stern M (1997) Total immunoreactive proinsulin, immunoreactive insulin and specific insulin in relation to conversion to NIDDM: the Mexico City Diabetes Study. Diabetologia 40:830-837

5. Bergman RN, Finegood DT, Kahn SE (2002) The evolution of beta-cell dysfunction and insulin resistance in type 2 diabetes. Eur J Clin Invest 32(Suppl 3):S35-S45

6. Mykkanen L, Haffner SM, Kuusisto J, Pyorala K, Hales CN, Laakso M (1995) Serum proinsulin levels are disproportionately increased in elderly prediabetic subjects. Diabetologia 38:11761182

7. Pradhan AD, Manson JE, Meigs JB et al (2003) Insulin, proinsulin, proinsulin:insulin ratio, and the risk of developing type 2 diabetes mellitus in women. Am J Med 114:438-444

8. Schulze MB, Solomon CG, Rifai N et al (2005) Hyperproinsulinaemia and risk of type 2 diabetes mellitus in women. Diabet Med 22:1178-1184

9. Kahn SE, Leonetti DL, Prigeon RL, Boyko EJ, Bergstrom RW, Fujimoto WY (1995) Proinsulin as a marker for the development of NIDDM in Japanese-American men. Diabetes 44:173-179

10. Hanley AJ, D’Agostino R Jr, Wagenknecht LE et al (2002) Increased proinsulin levels and decreased acute insulin response independently predict the incidence of type 2 diabetes in the insulin resistance atherosclerosis study. Diabetes 51:1263-1270

11. Zethelius B, Byberg L, Hales CN, Lithell H, Berne C (2003) Proinsulin and acute insulin response independently predict type 2 diabetes mellitus in men - report from 27 years of follow-up study. Diabetologia 46:20-26

12. Zethelius B, Hales CN, Lithell HO, Berne C (2004) Insulin resistance, impaired early insulin response, and insulin propeptides as predictors of the development of type 2 diabetes: a population-based, 7-year follow-up study in 70-year-old men. Diabetes Care 27:1433-1438

13. Nijpels G, Popp-Snijders C, Kostense PJ, Bouter LM, Heine RJ (1996) Fasting proinsulin and 2-h post-load glucose levels predict the conversion to NIDDM in subjects with impaired glucose tolerance: the Hoorn Study. Diabetologia 39:113-118

14. Wareham NJ, Byrne CD, Williams R, Day NE, Hales CN (1999) Fasting proinsulin concentrations predict the development of type 2 diabetes. Diabetes Care 22:262-270

15. Vauhkonen IK, Niskanen LK, Mykkanen L, Haffner SM, Uusitupa MI, Laakso M (2000) Hyperproinsulinemia is not a characteristic feature in the offspring of patients with different phenotypes of type II diabetes. Eur J Endocrinol 143:251-260

16. Bonora E, Zavaroni I, Coscelli C, Butturini U (1983) Decreased hepatic insulin extraction in subjects with mild glucose intolerance. Metabolism 32:438-446 
17. Wagenknecht LE, Mayer EJ, Rewers M et al (1995) The Insulin Resistance Atherosclerosis Study (IRAS) objectives, design, and recruitment results. Ann Epidemiol 5:464-472

18. World Health Organization (1999) Definition, diagnosis and classification of diabetes mellitus and its complications. Part 1: diagnosis and classification of diabetes mellitus

19. DeLong ER, DeLong DM, Clarke-Pearson DL (1988) Comparing the areas under two or more correlated receiver operating characteristic curves: a nonparametric approach. Biometrics 44:837-845

20. Weiss R, Caprio S, Trombetta M, Taksali SE, Tamborlane WV, Bonadonna R (2005) Beta-cell function across the spectrum of glucose tolerance in obese youth. Diabetes 54:1735-1743

21. Larsson H, Ahren B (1999) Relative hyperproinsulinemia as a sign of islet dysfunction in women with impaired glucose tolerance. J Clin Endocrinol Metab 84:2068-2074 